University of South Florida

DIGITAL COMMONS

Digital Commons @ University of

@ UNIVERSITY OF SOUTH FLORIDA

South Florida

$1-1-2011$

\title{
2011 Work Plan USF Polytechnic
}

USF

Follow this and additional works at: https://digitalcommons.usf.edu/usf_accountability_reports

\section{Scholar Commons Citation}

USF, "2011 Work Plan USF Polytechnic" (2011). USF Accountability Reports. 21.

https://digitalcommons.usf.edu/usf_accountability_reports/21

This Article is brought to you for free and open access by the USF Archives at Digital Commons @ University of South Florida. It has been accepted for inclusion in USF Accountability Reports by an authorized administrator of Digital Commons @ University of South Florida. For more information, please contact digitalcommons@usf.edu. 


\section{Update to the \\ University of South Florida Polytechnic Work Plan}


Note concerning data accuracy: The Office of the Board of Governors believes that the accuracy of the data it collects and reports is paramount to ensuring accountability in the State University System. Thus, the Board Office allows university resubmissions of some data to correct errors when they are discovered. This policy can lead to changes in historical data. 


\begin{tabular}{|c|c|c|c|c|c|c|c|}
\hline \multicolumn{8}{|c|}{ University of South Florida 2010 Annual Report } \\
\hline & & & \multicolumn{5}{|c|}{ USF Polytechnic } \\
\hline Enrollments & \# & $\%$ & \multicolumn{3}{|c|}{ Degree Programs Offered (As of Spr. 10) } & \multicolumn{2}{|c|}{ Carnegie Classification } \\
\hline $\begin{array}{c}\text { TOTAL } \\
\text { (Fall 2009) }\end{array}$ & 1,299 & $100 \%$ & \multicolumn{2}{|l|}{ TOTAL } & 16 & $\begin{array}{c}\text { Undergraduate } \\
\text { Instructional Program: }\end{array}$ & \multirow{10}{*}{$\begin{array}{c}\text { SEPARATE CLASSIFICATION } \\
\text { PENDING }\end{array}$} \\
\hline Black & 129 & $10 \%$ & \multicolumn{2}{|c|}{ Baccalaureate } & 11 & Graduate Instructional & \\
\hline Hispanic & 124 & $10 \%$ & \multicolumn{2}{|c|}{ Master's \& Specialist's } & 5 & Program: & \\
\hline White & 976 & $75 \%$ & \multicolumn{2}{|c|}{ Research Doctorate } & 0 & Enrollment Profile: & \\
\hline Other & 70 & $5 \%$ & \multicolumn{2}{|c|}{ Professional Doctorate } & 0 & Undergraduate Profile: & \\
\hline Full-Time & 509 & $39 \%$ & \multirow{2}{*}{$\begin{array}{l}\text { Faculty } \\
\text { (Fall 2009) }\end{array}$} & \multirow{2}{*}{$\begin{array}{l}\text { Full- } \\
\text { Time }\end{array}$} & \multirow{2}{*}{ Part-Time } & Size and Setting: & \\
\hline Part-Time & 790 & $61 \%$ & & & & \multirow{2}{*}{ Basic: } & \\
\hline Undergraduate & 1,055 & $81 \%$ & TOTAL & 30 & 5 & & \\
\hline Graduate & 201 & $15 \%$ & Tenure/T. Track & 19 & 0 & \multirow{2}{*}{ Elective Classification: } & \\
\hline Unclassified & 43 & $3 \%$ & Other Faculty/Instr. & 11 & 5 & & \\
\hline
\end{tabular}

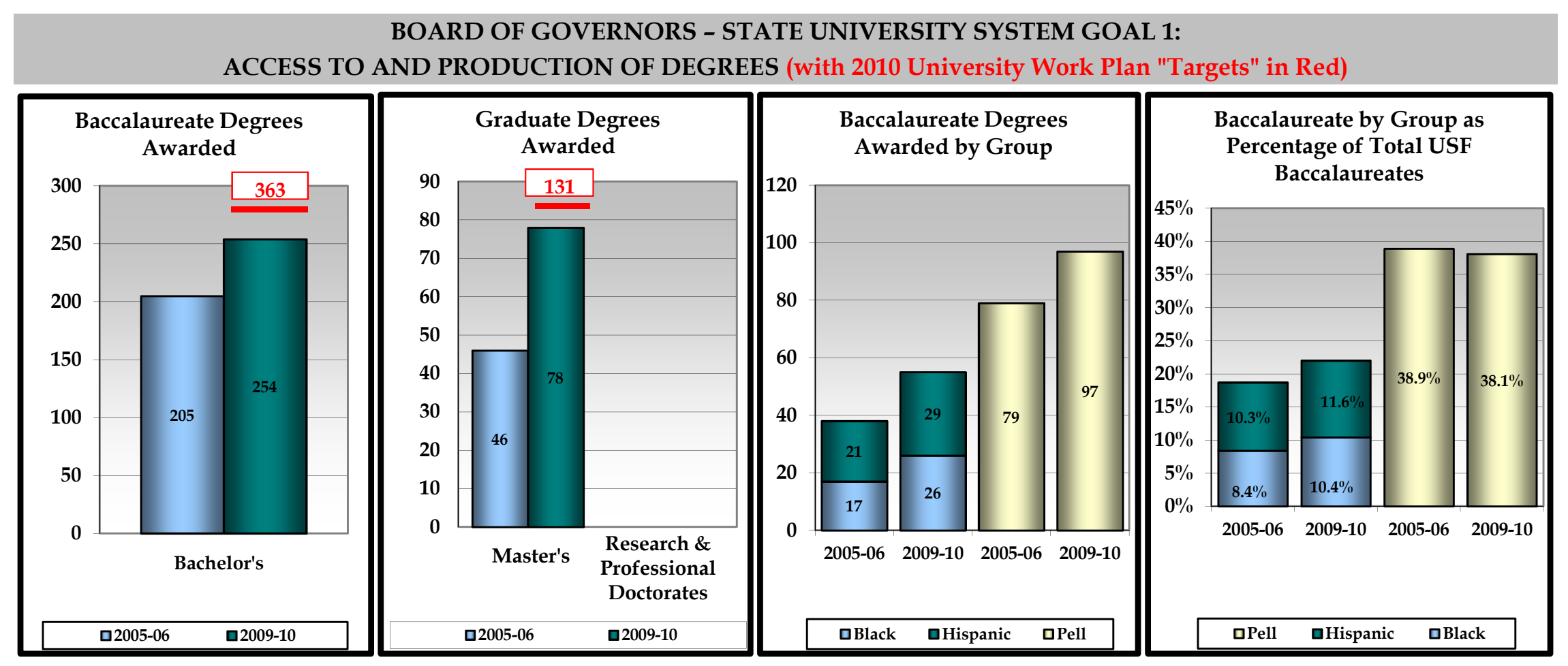

2012 - 2013 Projected Institutional Contributions in RED PRINT. 
BOARD OF GOVERNORS - STATE UNIVERSITY SYSTEM GOAL 2:

MEETING STATEWIDE PROFESSIONAL AND WORKFORCE NEEDS (with 2010 University Work Plan "Targets" in Red)

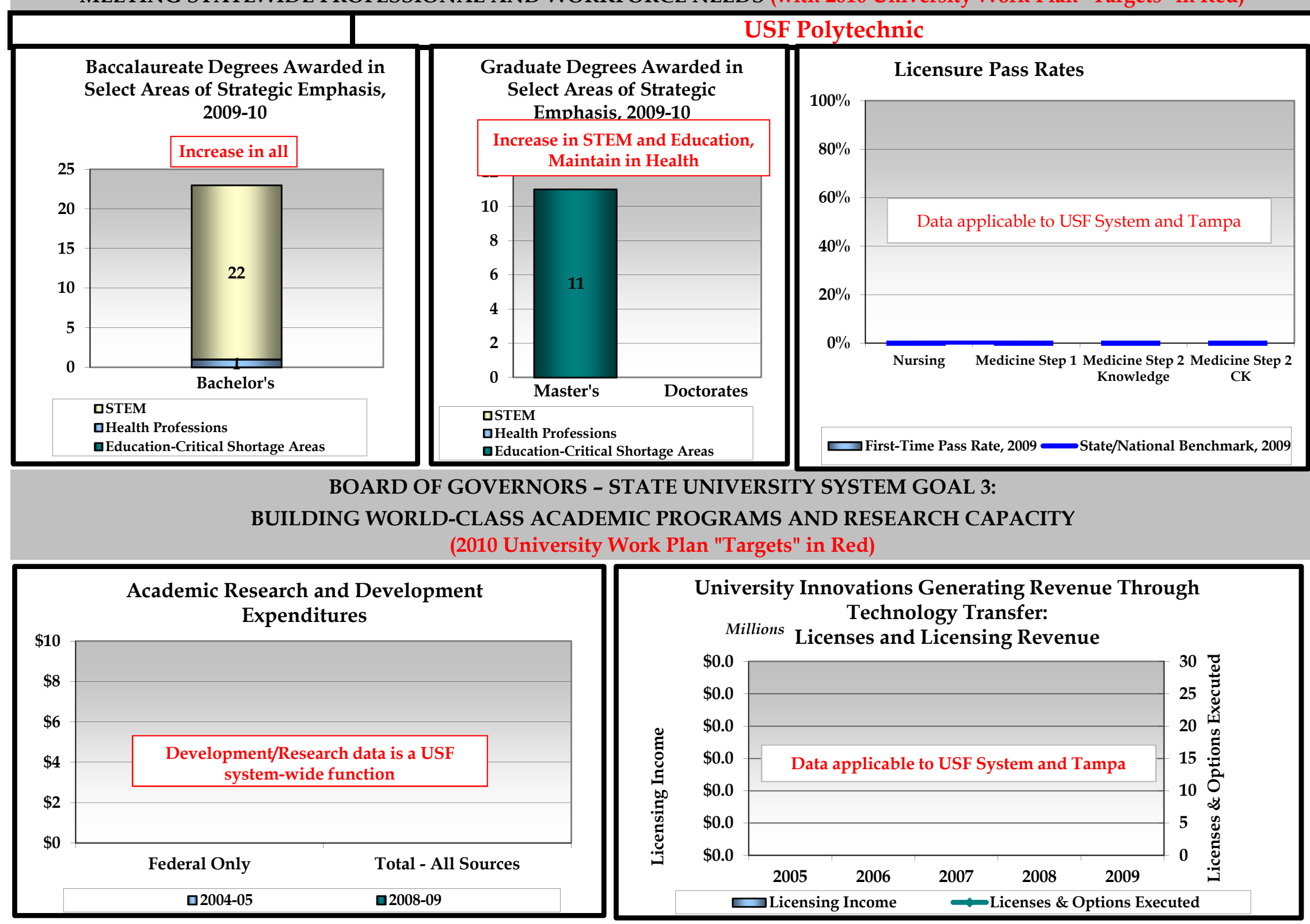

Projected Institutional Contributions in RED PRINT

(2012 - 2013 for TOTAL Degrees in Areas of Strategic Emphasis; 2012 for NCLEX; 2011 -2012 for R\&D, Licences, and Licensing Revenue). 


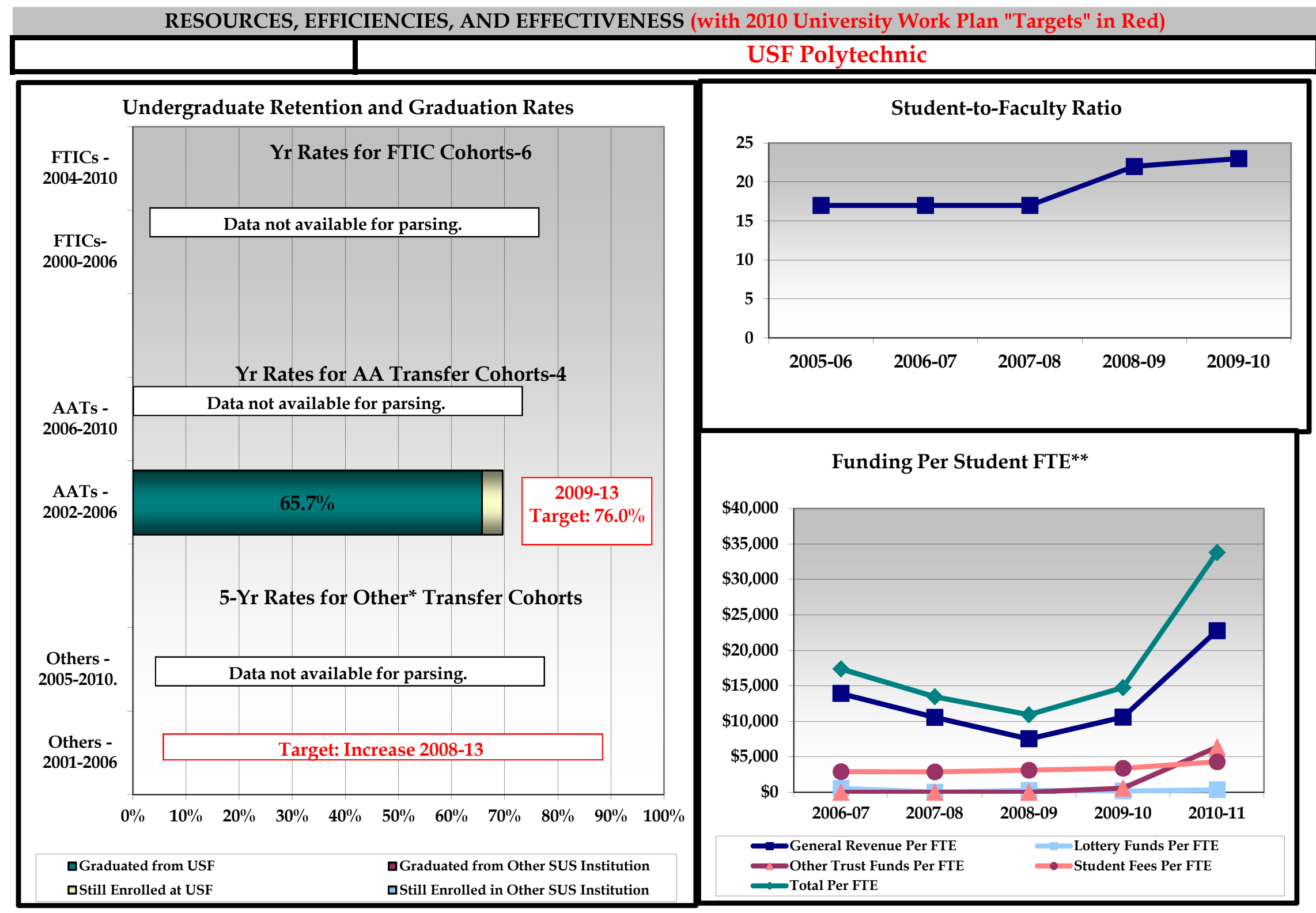

$*$ The composition of "Other Transfer" cohorts may vary greatly by institution
and by year. $\quad \begin{array}{r}* * \text { FTE for this metric uses the standard IPEDS definition of FTE, equal to } 30 \\ \text { credit hours for undergraduates and } 24 \text { for graduates. }\end{array}$ Graduation Rate from SAME Institution - Projected Institutional Contributions in RED PRINT. 


\section{Select Data Tables from the 2009-2010 Annual Report}

* Peer choices should be noted. In cases in which peer data are not available for a specific metric, but are available for a related metric,an institution might want to note such in the "Comparison with Peers" row.

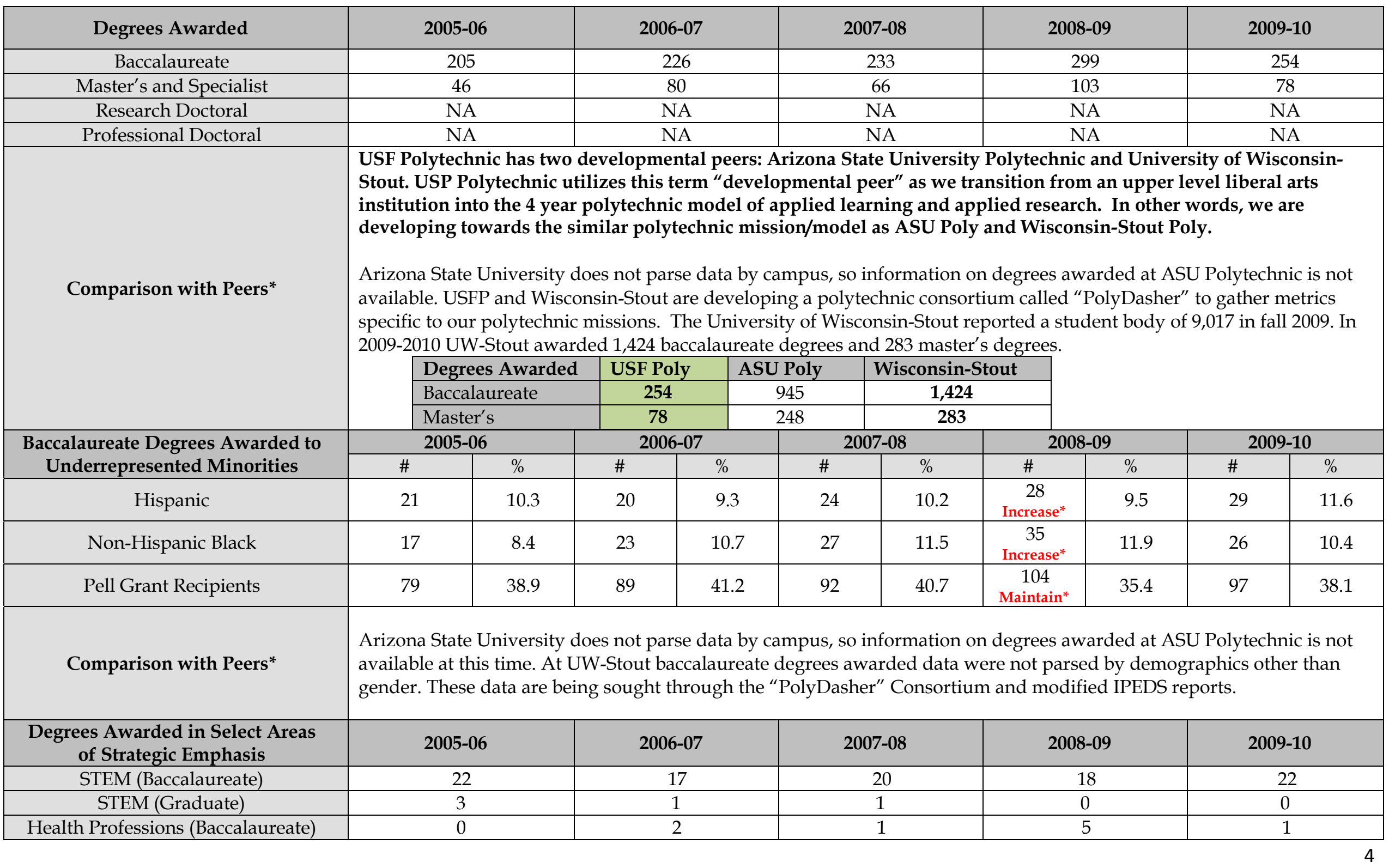


Health Professions (Graduate) Education-Critical Shortage (Bacc.) Education-Critical Shortage (Grad.)

\section{Comparison with Peers*}

Undergraduate Retention and
Graduation Rates from Same Institution

Fed.Def.: 6-Yr Rates Full-Time FTICs SUS Def.: 6-Yr Rates - FTICS

SUS Def.: 4-Yr Rates - AA Transfers SUS Def.: 5-Yr Rates - Others

\section{Comparison with Peers*}

\begin{tabular}{|c|c|c|c|}
\hline 0 & 0 & 2 & 0 \\
\hline 0 & 0 & 0 & 0 \\
\hline 19 & 22 & 28 & 11 \\
\hline
\end{tabular}

12

\begin{tabular}{|r|r|}
\hline 0 & 0 \\
\hline 0 & 0 \\
\hline 2 & 19
\end{tabular}

Arizona State University does not parse data by campus, so information on degrees awarded at ASU Polytechnic is not available. In 2009-2010 at UW-Stout 38 baccalaureate degrees and 6 master's degrees were awarded in Engineering; 117 baccalaureate degrees and 18 in Information Technology; and 27 baccalaureate degrees and 9 master's degrees in applied science. Of these degrees awarded by UW-Stout 24 baccalaureate and 20 master's degrees were in Health Professions, and 62 baccalaureate and 3 master's degrees were in Education-Critical Shortage areas.

\begin{tabular}{|c|l|l|l|l|l|l|l|l|l|}
\hline \multicolumn{2}{|c|}{ By 2006 } & \multicolumn{2}{c|}{ By 2007 } & \multicolumn{2}{c|}{ By 2008 } & \multicolumn{3}{c|}{ By 2009 } & \multicolumn{2}{c|}{ By 2010 } \\
\hline Grad & $\begin{array}{c}\text { Still } \\
\text { Enr }\end{array}$ & \multicolumn{1}{|c|}{ Grad } & $\begin{array}{c}\text { Still } \\
\text { Enr }\end{array}$ & \multicolumn{1}{|c|}{ Grad } & $\begin{array}{c}\text { Still } \\
\text { Enr }\end{array}$ & Grad & $\begin{array}{c}\text { Still } \\
\text { Enr }\end{array}$ & Grad & $\begin{array}{c}\text { Still } \\
\text { Enr }\end{array}$ \\
\hline NA & NA & NA & NA & NA & NA & NA & NA & NA & NA \\
\hline NA & NA & NA & NA & NA & NA & NA & NA & NA & NA \\
\hline
\end{tabular}

NA

\begin{tabular}{|c|c|c|c|c|c|}
\hline Licensure Exam Pass Rates & Year 1 & Year 2 & Year 3 & Year 4 & Year 5 \\
\hline Nursing (2005-06 Through 2009-10) & NA & NA & NA & NA & NA \\
\hline Medicine - Step 1 (2006 - 2010) & NA & NA & NA & NA & NA \\
\hline $\begin{array}{l}\text { Medicine - Step } 2 \text { Clinical } \\
\text { Knowledge } \\
\text { (2005-06 Through 2009-10) }\end{array}$ & NA & NA & NA & NA & NA \\
\hline $\begin{array}{l}\text { Medicine - Step } 2 \text { Clinical Skills } \\
(2005-06 \text { Through 2009-10) }\end{array}$ & NA & NA & NA & NA & NA \\
\hline
\end{tabular}

\section{Comparison with Peers*}

\begin{tabular}{|c|c|c|c|c|}
\hline $\begin{array}{c}\text { Academic Research and } \\
\text { Development Expenditures }\end{array}$ & $2004-05$ & $2005-06$ & $2006-07$ & $2007-08$ \\
\hline Federal Only (Thousand \$) & $\$$ & $\$$ & $\$$ & $\$$ \\
\hline Total - All Sources (Thousand \$) & $\$$ & $\$$ & $\$$ & $\$$ \\
\hline
\end{tabular}




\begin{tabular}{|l|l|}
\hline Comparison with Peers* & NA \\
\hline
\end{tabular}




\begin{tabular}{|c|c|c|c|c|c|}
\hline Technology Transfer & 2005 & 2006 & 2007 & 2008 & 2009 \\
\hline Licenses \& Options Executed & NA & NA & NA & NA & NA \\
\hline Licensing Income & NA & NA & NA & NA & NA \\
\hline Comparison with Peers* & NA & & & & \\
\hline \multicolumn{6}{|l|}{$\begin{array}{l}\text { OTHER KEY OUTPUT OR } \\
\text { OUTCOME METRICS }\end{array}$} \\
\hline & & & & & \\
\hline & & & & & \\
\hline & & & & & \\
\hline & & & & & \\
\hline & & & & & \\
\hline & & & & & \\
\hline & & & & & \\
\hline \multicolumn{6}{|l|}{ Comparison with Peers* } \\
\hline \multicolumn{6}{|c|}{$\begin{array}{c}\text { Based on Review of Data Trends on Key Output or Outcome Metrics Identified Here and/or in Annual Report, } \\
\text { Three (3) Areas of Concern/Areas Needing Improvement }\end{array}$} \\
\hline \multicolumn{6}{|c|}{$\begin{array}{l}\text { (1) Increase the number of baccalaureate degrees awarded. A SACS request for substantive change is in process for allowing a freshmen } \\
\text { cohort in } 2012 \text { and the first freshmen class in } 2013 \text { following SACS accreditation. The general education curriculum and faculty hires are } \\
\text { in process. In addition, new concentrations in applied science and general studies are coming on line now with additional baccalaureate } \\
\text { degrees under development following SACS accreditation. Significant effort towards recruitment, persistence and retention are underway } \\
\text { to serve our current pool of transfer students. }\end{array}$} \\
\hline \multicolumn{6}{|c|}{$\begin{array}{l}\text { (2) Increase the number of master's degrees awarded. The completion of approval of the M.S. in Information Technology is at the BOG level } \\
\text { with a plan implementation of fall } 2011 \text {. As with the baccalaureate degrees, additional faculty hires and curriculum development are in } \\
\text { process for implementation of new master's programs following SACS accreditation. }\end{array}$} \\
\hline
\end{tabular}


(3) Increase the number of baccalaureate degrees awarded in STEM fields. Goal 3 of the USFP Strategic Plan states: Expand and create academic programs that focus on applied learning, applied research, applied technology, and interdisciplinary approaches in a polytechnic model. Develop and implement new degree programs in five areas of distinction; applied health sciences; mathematics and science education; business and entrepreneurship; manufacturing engineering and technology; and information technologies (all STEM fields). As we develop our polytechnic model, all processes and procedures are focused on this commitment. 


\section{UPDATES TO 2010 UNIVERSITY WORK PLAN}

[Please identify briefly any critical changes only to information provided in the 2010 University Work Plan that was not updated in the 2009-2010 Annual Report regarding the institution's strategic plan; institutional mission, vision, and strategic directions for the next five to ten years; current or aspirational peer institutions; windows of opportunity; or unique challenges.]

\section{Change:}

The list of New Academic Degree Program Proposals over the next three years was updated from the 2010 list in the following ways:

- Degrees in Manufacturing Engineering and Manufacturing Technology have been removed and replaced with degrees in Systems Engineering (B). Refinement of focus to systems perspective provides for interdisciplinary opportunities with Innovation Management.

- Two new programs were added in Accounting \& Financial Management (B) and Health Information Management (B), in keeping with the development of the Innovation Incubators and emphasis on economic development needs of the Central Florida region and State.

\section{Significant updates:}

- The initial SACS Accreditation Application was submitted in December 2010. Targeted completion of accreditation by December 2012 is still anticipated.

- Implementation of the M.S. in Information Technology is expected for fall 2011.

- Twenty-two new faculty were hired for the 2010-2011 academic year; $14 \%$ received degrees from, or experience working, in a polytechnic university; 55\% had degrees from institutions classified as Very High Research Activity; and 18\% had degrees from institutions classified as High Research Activity. Thirty seven additional faculty hires are in process for 2011-12.

- Work on infrastructure for the new I-4 campus site began in fall 2010.

\section{Unique Challenges:}

- State College System: Increased competition and risk of duplication of programs/services within the higher education market.

\section{USF Polytechnic and the USF System:}

- USF Polytechnic is an integral part of the evolving USF System. It works closely with the other USF institutions to enhance the mission of the USF System and helps to facilitate the individual missions of all four institutions. Specific benefits include: enhanced access for students, distinctiveness while optimizing campus potential, greater choice to meet student and academic needs, broader advocacy, efficiencies (both academic and economic), commitment to meeting local needs, leveraging our combined strength through collaboration, and a unified brand yielding identity and impact. 
CAVP Academic Coordination Project (List degree programs recommended for new collaborative or joint delivery model or other corrective action, as well as any degree programs recommended for continuation but for which university and Board staff have not reached agreement on the sufficiency of the rationale.)

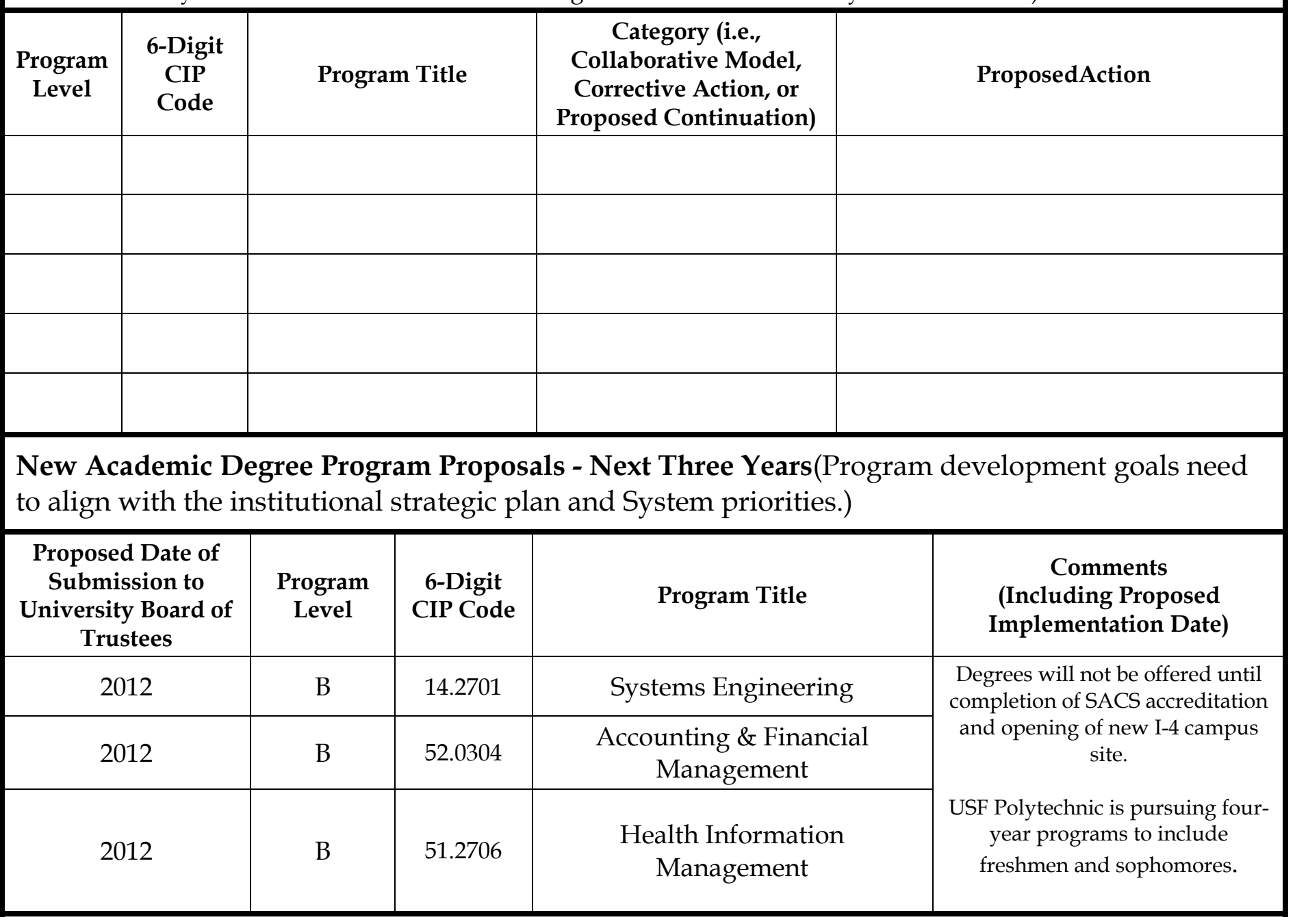




\section{Enrollment Planning}

Please explain briefly any planned changes in enrollment patterns in the next five years, with rationale (e.g., more emphasis on enrolling FCS AA transfers; enrollment of more out-of-state students; enrollment of more FTICs as the institution builds out a more residential experience for undergraduates; maintain undergraduate enrollment with more growth at graduate level to align with institutional mission; plan to maintain current enrollment with more emphasis on improving graduation rates; etc.).

- Increased lower-level course offerings, beginning fall 2011.

- Enrollment of a Pilot Freshman Cohort in 2012.

- Enrollment of a Freshman Class in 2013.

- Increased graduate enrollment with implementation of the M.S. in Information Technology in fall 2011.

1. Annual FTE enrollment plans by level, site, and residency for tuition purposes in the format provided in the template on the next pages.

2. These are only to include fundable FTE enrollments. So, for example, out-of-state profile admits should not be included in the out-of-state data.

3. Remember that Pharm.D., Law, and other Professional Doctorates (per the recently changed IPEDS definitions)should be counted asGrad II enrollments.

4. An explanation of over-enrollment is required for any level in which the 2010-11 funded enrollment plan lagged actual 2010-11 enrollment by more than 5\% (Section 1011.90, F.S.). 


\begin{tabular}{|c|c|c|c|c|c|c|c|c|}
\hline \multicolumn{9}{|c|}{$\begin{array}{l}\text { Enrollment Plan Proposal - All State-Fundable FTE Enrollments } \\
\text { (Except Medical/Dental/Veterinary Enrollments) POLYTECHNIC }\end{array}$} \\
\hline $\begin{array}{l}\text { For entire } \\
\text { institution }\end{array}$ & Funded & Estimated & Funded & Estimated & Estimated & Estimated & Estimated & \multirow{2}{*}{$\begin{array}{c}\text { 5-Year } \\
\text { Projected } \\
\text { Average } \\
\text { Annual } \\
\text { Growth } \\
\text { Rate } \\
\end{array}$} \\
\hline FTE & $2010-11$ & $2010-11$ & 2011-12 & 2011-12 & 2012-13 & 2014-15 & 2016-17 & \\
\hline $\begin{array}{c}\text { FL } \\
\text { Resident } \\
\text { Lower } \\
\end{array}$ & 0 & 56 & 0 & 56 & 228 & 305 & 418 & $129 \%$ \\
\hline $\begin{array}{c}\text { FL } \\
\text { Resident } \\
\text { Upper }\end{array}$ & 494 & 740 & 494 & 793 & 910 & 1,185 & 1,626 & $21 \%$ \\
\hline $\begin{array}{c}\text { FL } \\
\text { Resident } \\
\text { Grad I } \\
\end{array}$ & 103 & 94 & 103 & 129 & 139 & 146 & 195 & $10 \%$ \\
\hline $\begin{array}{c}\text { FL } \\
\text { Resident } \\
\text { Grad II }\end{array}$ & 0 & 0 & 0 & 0 & 0 & 0 & 0 & 0 \\
\hline $\begin{array}{l}\text { Total FL } \\
\text { Resident }\end{array}$ & 597 & 890 & 597 & 978 & 1,277 & 1,636 & 2,239 & $25.8 \%$ \\
\hline $\begin{array}{c}\text { Non-Res. } \\
\text { Lower }\end{array}$ & & 1 & & 3 & 8 & 10 & 22 & $127 \%$ \\
\hline $\begin{array}{c}\text { Non-Res. } \\
\text { Upper }\end{array}$ & & 9 & & 9 & 12 & 15 & 38 & $64 \%$ \\
\hline $\begin{array}{c}\text { Non-Res. } \\
\text { Grad I }\end{array}$ & & 0 & & 1 & 2 & 3 & 12 & $220 \%$ \\
\hline $\begin{array}{c}\text { Non-Res. } \\
\text { Grad II }\end{array}$ & & 0 & & 0 & 0 & 0 & 0 & 0 \\
\hline $\begin{array}{l}\text { Total Non- } \\
\text { Res. }\end{array}$ & 0 & 10 & 0 & 13 & 22 & 28 & 72 & $90.8 \%$ \\
\hline $\begin{array}{l}\text { Total } \\
\text { Lower }\end{array}$ & & 57 & & 59 & 236 & 315 & 440 & $129 \%$ \\
\hline $\begin{array}{c}\text { Total } \\
\text { Upper }\end{array}$ & & 749 & & 802 & 922 & 1,200 & 1,664 & $21 \%$ \\
\hline $\begin{array}{c}\text { Total Grad } \\
\text { I }\end{array}$ & & 94 & & 130 & 141 & 149 & 207 & $12 \%$ \\
\hline $\begin{array}{l}\text { Total Grad } \\
\text { II }\end{array}$ & & 0 & & 0 & 0 & 0 & 0 & 0 \\
\hline Total FTE & 597 & 900 & 597 & 991 & 1,299 & 1,664 & 2,311 & $26.6 \%$ \\
\hline
\end{tabular}




\begin{tabular}{|c|c|c|c|c|c|c|c|c|}
\hline \multicolumn{9}{|c|}{ Enrollment Plan Proposal - Medical/Dental/Veterinary State-FundableEnrollments } \\
\hline $\begin{array}{l}\text { For entire } \\
\text { institution }\end{array}$ & Funded & Estimated & Funded & Estimated & Estimated & Estimated & Estimated & \multirow{2}{*}{$\begin{array}{c}\text { 5-Year } \\
\text { Projected } \\
\text { Average } \\
\text { Annual } \\
\text { Growth } \\
\text { Rate }\end{array}$} \\
\hline Headcount & $2010-11$ & $2010-11$ & 2011-12 & 2011-12 & $2012-13$ & 2014-15 & 2016-17 & \\
\hline $\begin{array}{c}\text { FL Resident } \\
\text { Medical } \\
\text { Headcount }\end{array}$ & & & & & & & & \\
\hline $\begin{array}{l}\text { Non-Res. } \\
\text { Medical } \\
\text { Headcount }\end{array}$ & & & & & & & & \\
\hline \multicolumn{9}{|c|}{$\begin{array}{l}\text { Total Medical } \\
\text { Headcount }\end{array}$} \\
\hline \multicolumn{9}{|l|}{$\begin{array}{c}\text { FL Resident } \\
\text { Dentistry } \\
\text { Headcount }\end{array}$} \\
\hline \multicolumn{9}{|l|}{$\begin{array}{l}\text { Non-Res. } \\
\text { Dentistry } \\
\text { Headcount }\end{array}$} \\
\hline \multicolumn{9}{|l|}{$\begin{array}{c}\text { Total } \\
\text { Dentistry } \\
\text { Headcount }\end{array}$} \\
\hline \multicolumn{9}{|l|}{$\begin{array}{c}\text { FL Resident } \\
\text { Veterinary } \\
\text { Headcount }\end{array}$} \\
\hline \multicolumn{9}{|l|}{$\begin{array}{l}\text { Non-Res. } \\
\text { Veterinary } \\
\text { Headcount }\end{array}$} \\
\hline $\begin{array}{c}\text { Total } \\
\text { Veterinary } \\
\text { Headcount }\end{array}$ & & & & & & & & \\
\hline
\end{tabular}

[This medical headcount is MD-only, not all HSC enrollments.] 
For each distinct physical location (main, branch, site, regional campus) that has or is planned to have more than 150 FTEState-fundableenrollments

\section{SITE:}

\begin{tabular}{|c|c|c|c|c|c|c|}
\hline & Estimated & Estimated & Estimated & Estimated & Estimated & \multirow{2}{*}{$\begin{array}{c}\text { 5-Year } \\
\text { Projected } \\
\text { Average } \\
\text { Annual } \\
\text { Growth Rate }\end{array}$} \\
\hline FTE & $2010-11$ & 2011-12 & 2012-13 & 2014-15 & 2016-17 & \\
\hline Lower & & & & & & \\
\hline Upper & & & & & & \\
\hline Grad I & & & & & & \\
\hline \multicolumn{7}{|l|}{ Grad II } \\
\hline \multicolumn{7}{|l|}{ Total } \\
\hline \multicolumn{7}{|l|}{ SITE: } \\
\hline & Estimated & Estimated & Estimated & Estimated & Estimated & \multirow{2}{*}{$\begin{array}{c}\text { 5-Year } \\
\text { Projected } \\
\text { Average } \\
\text { Annual } \\
\text { Growth Rate }\end{array}$} \\
\hline FTE & $2010-11$ & 2011-12 & 2012-13 & 2014-15 & 2016-17 & \\
\hline \multicolumn{7}{|l|}{ Lower } \\
\hline \multicolumn{7}{|l|}{ Upper } \\
\hline \multicolumn{7}{|l|}{ Grad I } \\
\hline \multicolumn{7}{|l|}{ Grad II } \\
\hline \multicolumn{7}{|l|}{ Total } \\
\hline \multicolumn{7}{|l|}{ SITE: } \\
\hline & Estimated & Estimated & Estimated & Estimated & Estimated & \multirow{2}{*}{$\begin{array}{c}\text { 5-Year } \\
\text { Projected } \\
\text { Average } \\
\text { Annual } \\
\text { Growth Rate } \\
\end{array}$} \\
\hline FTE & $2010-11$ & 2011-12 & 2012-13 & 2014-15 & 2016-17 & \\
\hline \multicolumn{7}{|l|}{ Lower } \\
\hline \multicolumn{7}{|l|}{ Upper } \\
\hline \multicolumn{7}{|l|}{ Grad I } \\
\hline \multicolumn{7}{|l|}{ Grad II } \\
\hline Total & & & & & & \\
\hline
\end{tabular}


For the sum of the remaining physical locations with fewer than 150 current or planned State-fundableFTE enrollments.

SITE: REMAINING PHYSICAL LOCATIONS: Citrus County and Avon Park (Elem. Ed, Ed. Leadership)

\begin{tabular}{|c|c|c|c|c|c|c|}
\hline & Estimated & Estimated & Estimated & Estimated & Estimated & $\begin{array}{c}\text { 5-Year } \\
\text { Projected } \\
\text { Average } \\
\text { Annual } \\
\text { Growth Rate }\end{array}$ \\
\hline Lower & $\mathbf{2 0 1 0 - 1 1}$ & $\mathbf{2 0 1 1 - 1 2}$ & $\mathbf{2 0 1 2 - 1 3}$ & $\mathbf{2 0 1 4 - 1 5}$ & $\mathbf{2 0 1 6 - 1 7}$ & 0 \\
\hline Upper & 0 & 0 & 0 & 0 & 0 & $3.5 \%$ \\
\hline Grad I & 29.8 & 34 & 35 & 38 & 40 & $12.7 \%$ \\
\hline Grad II & 10.1 & 11 & 12 & 15 & 18 & 0 \\
\hline Total & 0 & 0 & 0 & 0 & 0 & $5.8 \%$ \\
\hline
\end{tabular}

For the sum of current or planned State-fundableFTE enrollments not served at a physical location.

- SITE: VIRTUAL INSTRUCTION/DISTANCE LEARNING POLYTECHNIC

\begin{tabular}{|c|c|c|c|c|c|c|}
\hline & Estimated & Estimated & Estimated & Estimated & Estimated & \\
\hline FTE & $2010-11$ & 2011-12 & $2012-13$ & 2014-15 & 2016-17 & $\begin{array}{c}\text { Average } \\
\text { Annual } \\
\text { Growth Rate }\end{array}$ \\
\hline Lower & & & & & & \\
\hline Upper & & & & & & \\
\hline Grad I & & & & & & \\
\hline Grad II & & & & & & \\
\hline Total & & & & & & \\
\hline
\end{tabular}


Primary Institutional Goals/Metrics for the Next One to Three Years(In the context of the institutional strategic plan and vision, as well as System priorities, present three (3) to five (5) goals on which university effort will be focused in the next one to three years. Describe each goal, including whether the goal is new or continuing, the strategies for achieving that goal, the timeline and metrics by which success will be measured, expected outcomes, and assumptions, including financial, upon which the projected outcomes are predicated.) Each university is asked to include one goal associated with improved baccalaureate retention and graduation (e.g., improved first-year retention; reduce attainment gaps for underrepresented groups; improve graduation rates for AA transfers; etc.).

\begin{tabular}{|c|c|c|c|c|c|c|c|c|c|}
\hline \multicolumn{3}{|c|}{$\begin{array}{c}\text { Institutional Goal } \\
\text { [Indicate whether NEW or CONTINUING] }\end{array}$} & \multicolumn{3}{|c|}{ Implementation Strategies } & \multicolumn{4}{|c|}{ Metric(s)/Timeline/Expected Outcomes } \\
\hline \multicolumn{3}{|c|}{$\begin{array}{l}\text { \#1(Required) - Improve baccalaureate } \\
\text { retention and graduation. } \\
\text { (CONTINUING) }\end{array}$} & \multicolumn{3}{|c|}{$\begin{array}{l}\text { - Continue implementation of } \\
\text { Hobson's Communication and } \\
\text { Retention management systems. } \\
\text { - Increase the number of academic } \\
\text { advisors to provide enhanced service } \\
\text { to undergraduate students. } \\
\text { - Continue to monitor course offerings } \\
\text { to ensure scheduling of courses } \\
\text { required for majors and degree } \\
\text { completion. }\end{array}$} & \multicolumn{4}{|c|}{$\begin{array}{l}\text { - Full implementation expected by December } \\
\text { 2011. Retention reports will inform and assist } \\
\text { interventions. } \\
\text { - Expect full complement of advisors by spring } \\
2012 \text { (2 vacancies in process \& } 2 \text { additional in } \\
\text { hiring plan). } \\
\text { - Weekly enrollment management sessions } \\
\text { (beginning } 5 \text { weeks out from term) to monitor } \\
\text { and initiate immediate changes to enhance } \\
\text { enrollment and process improvements. } \\
\text { Expected Outcomes/Timeline: By 2014-15, a 3\% } \\
\text { increase in retention is expected with graduation } \\
\text { rate increasing by } 4 \% \text {. }\end{array}$} \\
\hline \multicolumn{4}{|c|}{ Proposed Funding Source: $2011-12$} & \multicolumn{6}{|c|}{ Proposed Funding Source: $2012-13$} \\
\hline $\begin{array}{l}\text { State/ Tuition } \\
\text { Revenue (est.) }\end{array}$ & $\begin{array}{l}\text { Other } \\
\text { (Identify } \\
\text { Revenue } \\
\text { Source - } \\
\text { e.g., Private) }\end{array}$ & $\begin{array}{l}\text { Undergrad. } \\
\text { Tuition } \\
\text { Differential } \\
\text { Revenue } \\
\text { (est.) }\end{array}$ & $\begin{array}{l}\text { Total from } \\
2011-12\end{array}$ & $\begin{array}{l}\text { Undergrad. } \\
\text { Tuition } \\
\text { Differential } \\
\text { Revenue } \\
\text { (est.) }\end{array}$ & $\begin{array}{l}\text { Legislative } \\
\text { Budget } \\
\text { Request } \\
\text { (State Funds) }\end{array}$ & $\begin{array}{l}\text { State/ } \\
\text { Tuition } \\
\text { Revenue } \\
\text { (est.) }\end{array}$ & $\begin{array}{c}\text { Other } \\
\text { (Identify } \\
\text { Revenue } \\
\text { Source - } \\
\text { e.g., } \\
\text { Private) } \\
\end{array}$ & $\begin{array}{l}\text { Total from } \\
\text { 2012-13 }\end{array}$ & $\begin{array}{l}2012-13 \text { to } \\
2016-17 \\
\text { PECO/ } \\
\text { Courtelis } \\
\text { Request }\end{array}$ \\
\hline & & $\$ 185,543$ & $\$ 185,543$ & $\$ 200,385$ & & & & $\$ 200,385$ & \\
\hline \multicolumn{3}{|c|}{$\begin{array}{c}\text { Institutional Goal } \\
\text { [Indicate whether NEW or CONTINUING] }\end{array}$} & \multicolumn{3}{|c|}{ Implementation Strategies } & \multicolumn{4}{|c|}{ Expected Outcomes/Metric(s)/Timeline } \\
\hline \multicolumn{3}{|c|}{$\begin{array}{l}\text { \#2 (Required) - STEM education and } \\
\text { engineering; interdisciplinary with business } \\
\text { and innovation management, and applied } \\
\text { research in Alternative Energy and Biofuels } \\
\text { Technologies (CONTINUING) }\end{array}$} & \multicolumn{3}{|c|}{$\begin{array}{l}\text { Hire Experienced Faculty to Develop } \\
\text { an Interdisciplinary Engineering } \\
\text { Degree Program and Establish a } \\
\text { Center for Applied Research in } \\
\text { Alternative Energy and Biofuels } \\
\text { Technologies }\end{array}$} & \multicolumn{4}{|c|}{$\begin{array}{l}\text { - Faculty will have identified talent and capacity } \\
\text { to deliver the polytechnic mission: } \\
\text { interdisciplinary and applied learning; } \\
\text { application in cutting-edge research and } \\
\text { technology to real world needs; and } \\
\text { collaborative partnerships that support }\end{array}$} \\
\hline
\end{tabular}




\begin{tabular}{|c|c|c|c|c|c|c|c|c|c|}
\hline & & & \multicolumn{3}{|c|}{$\begin{array}{l}\text { - Hire experienced faculty to develop a } \\
\text { degree program and establish an } \\
\text { applied research center that aligns } \\
\text { with critical needs identified in the } \\
\text { SUS Strategic Plan: STEM education } \\
\text { and engineering, with potential } \\
\text { further interdisciplinary opportunity } \\
\text { in business and innovation } \\
\text { management. } \\
\text { To enhance capacity to move quickly } \\
\text { to deliver new academic programs } \\
\text { and expand research initiatives } \\
\text { subsequent to separate SACS } \\
\text { accreditation. } \\
\text { Expand and/or create academic } \\
\text { programs that focus on applied } \\
\text { learning, applied research, applied } \\
\text { technology, and interdisciplinary } \\
\text { approaches in a polytechnic model. }\end{array}$} & \multicolumn{4}{|c|}{ 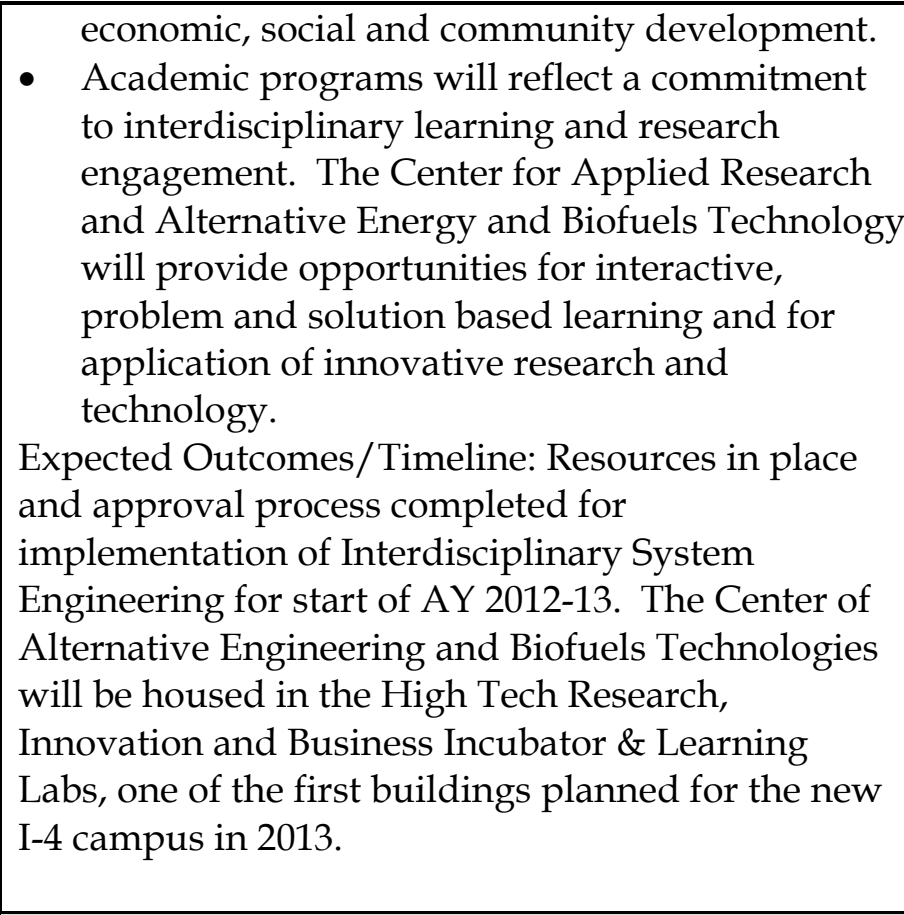 } \\
\hline \multicolumn{4}{|c|}{ Proposed Funding Source: 2011-12 } & \multicolumn{6}{|c|}{ Proposed Funding Source: $2012-13$} \\
\hline $\begin{array}{l}\text { State/ Tuition } \\
\text { Revenue (est.) }\end{array}$ & $\begin{array}{c}\text { Other } \\
\text { (Identify } \\
\text { Revenue } \\
\text { Source - } \\
\text { e.g., Private) }\end{array}$ & $\begin{array}{l}\text { Undergrad. } \\
\text { Tuition } \\
\text { Differential } \\
\text { Revenue } \\
\text { (est.) }\end{array}$ & $\begin{array}{l}\text { Total from } \\
2011-12\end{array}$ & $\begin{array}{l}\text { Undergrad } \\
\text { Tuition } \\
\text { Differential } \\
\text { Revenue } \\
\text { (est.) }\end{array}$ & \begin{tabular}{|} 
Legislative \\
Budget \\
Request \\
(State Funds)
\end{tabular} & $\begin{array}{l}\text { State/ } \\
\text { Tuition } \\
\text { Revenue } \\
\text { (est.) }\end{array}$ & $\begin{array}{c}\text { Other } \\
\text { (Identify } \\
\text { Revenue } \\
\text { Source - } \\
\text { e.g., } \\
\text { Private) } \\
\end{array}$ & $\begin{array}{l}\text { Total from } \\
2012-13\end{array}$ & $\begin{array}{l}\text { 2012-13 to } \\
2016-17 \\
\text { PECO/ } \\
\text { Courtelis } \\
\text { Request }\end{array}$ \\
\hline & & & & & $\$ 1,632,567$ & & & $\$ 1,632,567$ & \\
\hline \multicolumn{3}{|c|}{$\begin{array}{c}\text { Institutional Goal } \\
\text { [Indicate whether NEW or CONTINUING] }\end{array}$} & \multicolumn{3}{|c|}{ Implementation Strategies } & \multicolumn{4}{|c|}{ Expected Outcomes/Metric(s)/Timeline } \\
\hline \multicolumn{3}{|c|}{$\begin{array}{l}\# 3 \text { (Required) - New / Enhanced Degree } \\
\text { Programs, Faculty resources and support } \\
\text { requirements (NEW) }\end{array}$} & \multicolumn{3}{|c|}{$\begin{array}{l}\text { - Establish a faculty hiring plan that } \\
\text { includes faculty to enhance } \\
\text { Innovation and Technology } \\
\text { Programs, Education Programs, and } \\
\text { Human and Social Sciences Programs } \\
\text { for AY 2012-2013 searches. } \\
\text { - Provide the support required for } \\
\text { curriculum and delivery of academic }\end{array}$} & \multicolumn{4}{|c|}{$\begin{array}{l}\text { USF Polytechnic's academic structure will } \\
\text { enable USF Polytechnic (following USF System } \\
\text { degree and approval procedures and SACS and } \\
\text { Board of Governors notification and approval } \\
\text { requirements) to develop new degree programs } \\
\text { in a polytechnic model, including programs in } \\
\text { identified economic development industry } \\
\text { sectors and in the five areas of distinction }\end{array}$} \\
\hline
\end{tabular}




\begin{tabular}{|c|c|c|c|c|c|c|c|c|c|}
\hline & & & \multicolumn{3}{|c|}{ programs. } & \multicolumn{4}{|c|}{$\begin{array}{l}\text { identified in Goal } 3 \text { of the USFP Strategic Plan. } \\
\text { Expected Outcomes/Timeline: Following the SACS } \\
\text { accreditation (late 2012) and the degree program } \\
\text { approval process, new degree programs within the } \\
\text { Polytechnic model will assist in meeting the } \\
\text { identified economic development industry sectors. }\end{array}$} \\
\hline \multicolumn{4}{|c|}{ Proposed Funding Source: $2011-12$} & \multicolumn{6}{|c|}{ Proposed Funding Source: $2012-13$} \\
\hline $\begin{array}{l}\text { State/ Tuition } \\
\text { Revenue (est.) }\end{array}$ & $\begin{array}{l}\text { Other } \\
\text { (Identify } \\
\text { Revenue } \\
\text { Source - } \\
\text { e.g., Private) }\end{array}$ & $\begin{array}{l}\text { Undergrad } \\
\text { Tuition } \\
\text { Differential } \\
\text { Revenue } \\
\text { (est.) }\end{array}$ & $\begin{array}{l}\text { Total from } \\
\text { 2011-12 }\end{array}$ & $\begin{array}{l}\text { Undergrad } \\
\text { Tuition } \\
\text { Differential } \\
\text { Revenue } \\
\text { (est.) }\end{array}$ & $\begin{array}{c}\text { Legislative } \\
\text { Budget } \\
\text { Request } \\
\text { (State Funds) }\end{array}$ & $\begin{array}{c}\text { State/ } \\
\text { Tuition } \\
\text { Revenue } \\
\text { (est.) }\end{array}$ & $\begin{array}{c}\text { Other } \\
\text { (Identify } \\
\text { Revenue } \\
\text { Source - } \\
\text { e.g., } \\
\text { Private) } \\
\end{array}$ & $\begin{array}{c}\text { Total from } \\
\text { 2012-13 }\end{array}$ & $\begin{array}{l}2012-13 \text { to } \\
2016-17 \\
\text { PECO/ } \\
\text { Courtelis } \\
\text { Request }\end{array}$ \\
\hline \multicolumn{3}{|c|}{$\begin{array}{c}\text { Institutional Goal } \\
\text { [Indicate whether NEW or CONTINUING] }\end{array}$} & \multicolumn{3}{|c|}{ Implementation Strategies } & \multicolumn{4}{|c|}{ Expected Outcomes/Metric(s)/Timeline } \\
\hline \multicolumn{3}{|c|}{$\begin{array}{l}\# 4 \text { - Continue strategic hiring, general } \\
\text { education curriculum and academic } \\
\text { program development for implementation } \\
\text { of a Freshman Pilot Cohort in } 2012 \text { and a } \\
\text { Freshman Class in 2013. (CONTINUING) }\end{array}$} & \multicolumn{3}{|c|}{$\begin{array}{l}\text { - Establish a faculty hiring plan that } \\
\text { includes faculty in general education } \\
\text { content areas for AY 2011-2012 } \\
\text { searches. } \\
\text { - Establish a faculty committee for the } \\
\text { development of the general } \\
\text { education curriculum, complete } \\
\text { curriculum development and } \\
\text { approval processes. } \\
\text { Establish a First Year Experiences } \\
\text { Committee to identify facilities and } \\
\text { student services and supports } \\
\text { needed for implementation of the } \\
\text { Freshman Pilot Cohort in } 2012 \text { and } \\
\text { transition to a Freshman Class in } \\
\text { 2013. } \\
\text { Develop and implement a freshmen } \\
\text { recruitment and marketing plan. }\end{array}$} & \multicolumn{4}{|c|}{$\begin{array}{l}\text { - Some general education faculty already hired } \\
\text { with plans to add required full time faculty in } \\
\text { 2011-12 (pending budget). } \\
\text { - Faculty committee will have cohort gen ed } \\
\text { complete by May } 2011 \text { and } 2013 \text { class complete } \\
\text { by December } 2011 \text {. Will monitor progress } \\
\text { through the approval process. } \\
\text { - Committee began work in February } 2011 \text { with } \\
\text { ongoing process planning through early spring } \\
\text { 2012. Anticipate early admission for } 2012 \text { pilot. } \\
\text { Committee works from a project planning } \\
\text { document that incorporates action steps and } \\
\text { target expectations. } \\
\text { Plan is constantly monitored against } \\
\text { expectations. } \\
\text { Expected Outcomes: To have the resources in place } \\
\text { to implement the Freshmen Pilot Cohort in } 2012 \text { and } \\
\text { the Freshmen Class in } 2013 \text {. }\end{array}$} \\
\hline \multicolumn{4}{|c|}{ Proposed Funding Source: $2011-12$} & \multicolumn{6}{|c|}{ Proposed Funding Source: $2012-13$} \\
\hline
\end{tabular}




\begin{tabular}{|c|c|c|c|c|c|c|c|c|c|}
\hline $\begin{array}{l}\text { State/ Tuition } \\
\text { Revenue (est.) }\end{array}$ & $\begin{array}{c}\text { Other } \\
\text { (Identify } \\
\text { Revenue } \\
\text { Source - } \\
\text { e.g., Private) }\end{array}$ & $\begin{array}{c}\text { Undergrad } \\
\text { Tuition } \\
\text { Differential } \\
\text { Revenue } \\
\text { (est.) }\end{array}$ & $\begin{array}{l}\text { Total from } \\
2011-12\end{array}$ & $\begin{array}{l}\text { Undergrad } \\
\text { Tuition } \\
\text { Differential } \\
\text { Revenue } \\
\text { (est.) }\end{array}$ & $\begin{array}{l}\text { Legislative } \\
\text { Budget } \\
\text { Request } \\
\text { (State Funds) }\end{array}$ & $\begin{array}{l}\text { State/ } \\
\text { Tuition } \\
\text { Revenue } \\
\text { (est.) }\end{array}$ & $\begin{array}{c}\text { Other } \\
\text { (Identify } \\
\text { Revenue } \\
\text { Source - } \\
\text { e.g., } \\
\text { Private) } \\
\end{array}$ & $\begin{array}{l}\text { Total from } \\
2012-13\end{array}$ & $\begin{array}{c}2012-13 \text { to } \\
2016-17 \\
\text { PECO/ } \\
\text { Courtelis } \\
\text { Request }\end{array}$ \\
\hline$\$ 4,125,000$ & & & $\$ 4,125,000$ & & & $\$ 4,405,000$ & & $\$ 4,405,000$ & \\
\hline \multicolumn{3}{|c|}{$\begin{array}{l}\text { Institutional Goal } \\
\text { hether NEW or CONTINUING] }\end{array}$} & \multicolumn{3}{|c|}{ Implementation Strategies } & \multicolumn{4}{|c|}{ Expected Outcomes/Metric(s)/Timeline } \\
\hline \multicolumn{3}{|c|}{$\begin{array}{l}\text { \#5 - Complete separate SACS accreditation } \\
\text { and develop revised or new degree } \\
\text { programs for implementation in 2012-2013. } \\
\text { (CONINUING) }\end{array}$} & \multicolumn{3}{|c|}{$\begin{array}{l}\text { - Complete Compliance Certification } \\
\text { and SACS Accreditation site visit in } \\
\text { 2012. } \\
\text { Establish program development } \\
\text { committees in academic divisions to } \\
\text { complete degree program revisions } \\
\text { and/or new program proposals by } \\
\text { December 2011, and complete } \\
\text { program approval processes by } \\
\text { March 2012. } \\
\text { Develop new degree program } \\
\text { marketing and recruitment materials } \\
\text { by March 2012. } \\
\text { Complete new faculty hires where } \\
\text { needed for implementation of revised } \\
\text { or new degree programs by June } \\
\text { 2012 for start in August 2012. }\end{array}$} & \multicolumn{4}{|c|}{$\begin{array}{l}\text { - } \quad \text { Accreditation approval by December } 2012 . \\
\text { - } \quad \text { Meet expected timeline with close coordination } \\
\text { - } \quad \text { Recruitment materials will meet expected } \\
\text { timeline and strategy. } \\
\text { - } 22 \text { new faculty hired in } 2009-10 \text { and an } \\
\text { additional } 37 \text { searches in process for hire. } \\
\text { Additional hires will be dependent upon } \\
\text { resources. } \\
\text { Expected Outcomes: SACS accreditation in late } \\
\text { 2012 with new degree programs ready for } \\
\text { implementation in 2012-13. }\end{array}$} \\
\hline \multicolumn{4}{|c|}{ Proposed Funding Source: 2011-12 } & \multicolumn{6}{|c|}{ Proposed Funding Source: 2012-13 } \\
\hline $\begin{array}{l}\text { State/ Tuition } \\
\text { Revenue (est.) }\end{array}$ & $\begin{array}{c}\text { Other } \\
\text { (Identify } \\
\text { Revenue } \\
\text { Source - } \\
\text { e.g., Private) }\end{array}$ & $\begin{array}{l}\text { Undergrad } \\
\text { Tuition } \\
\text { Differential } \\
\text { Revenue } \\
\text { (est.) }\end{array}$ & $\begin{array}{l}\text { Total from } \\
2011-12\end{array}$ & $\begin{array}{l}\text { Undergrad } \\
\text { Tuition } \\
\text { Differential } \\
\text { Revenue } \\
\text { (est.) }\end{array}$ & $\begin{array}{l}\text { Legislative } \\
\text { Budget } \\
\text { Request } \\
\text { (State Funds) }\end{array}$ & $\begin{array}{l}\text { State/ } \\
\text { Tuition } \\
\text { Revenue } \\
\text { (est.) }\end{array}$ & $\begin{array}{c}\text { Other } \\
\text { (Identify } \\
\text { Revenue } \\
\text { Source - } \\
\text { e.g., } \\
\text { Private) } \\
\end{array}$ & $\begin{array}{l}\text { Total from } \\
2012-13\end{array}$ & $\begin{array}{c}2012-13 \text { to } \\
2016-17 \\
\text { PECO/ } \\
\text { Courtelis } \\
\text { Request }\end{array}$ \\
\hline$\$ 70,000$ & & & $\$ 70,000$ & & & $\$ 60,000$ & & $\$ 60,000$ & \\
\hline
\end{tabular}


OPTIONAL: Universities may add one or two additional goals.

\section{SUMMARY OF PROPOSED FUNDING FOR PRIMARY GOALS}

Proposed Funding Source: 2011-12

Proposed Funding Source: 2012-13

\begin{tabular}{|c|c|c|c|c|c|c|c|c|c|c|}
\hline Goal \# & $\begin{array}{c}\text { State/ } \\
\text { Tuition } \\
\text { Revenue } \\
\text { (est.) }\end{array}$ & $\begin{array}{c}\text { Other } \\
\text { (Identify } \\
\text { Revenue } \\
\text { Source - } \\
\text { e.g., } \\
\text { Private) } \\
\end{array}$ & $\begin{array}{l}\text { Undergrad } \\
\text { Tuition } \\
\text { Differential } \\
\text { Revenue } \\
\text { (est.) }\end{array}$ & $\begin{array}{l}\text { Total from } \\
2011-12\end{array}$ & $\begin{array}{l}\text { Undergrad } \\
\text { Tuition } \\
\text { Differential } \\
\text { Revenue } \\
\text { (est.) }\end{array}$ & $\begin{array}{l}\text { Legislative } \\
\text { Budget } \\
\text { Request } \\
\text { (State } \\
\text { Funds) }\end{array}$ & $\begin{array}{c}\text { State/ } \\
\text { Tuition } \\
\text { Revenue } \\
\text { (est.) }\end{array}$ & $\begin{array}{c}\text { Other } \\
\text { (Identify } \\
\text { Revenue } \\
\text { Source - } \\
\text { e.g., } \\
\text { Private) } \\
\end{array}$ & $\begin{array}{c}\text { Total from } \\
2012-13\end{array}$ & $\begin{array}{c}2012-13 \text { to } \\
2016-17 \\
\text { PECO/ } \\
\text { Courtelis } \\
\text { Request }\end{array}$ \\
\hline 1 & & & $\$ 185,543$ & $\$ 185,543$ & $\$ 200,385$ & & & & $\$ 200,385$ & \\
\hline 2 & & & & & & $\$ 1,632,567$ & & & $\$ 1,632,567$ & \\
\hline 3 & & & & & & & & & & \\
\hline $\begin{array}{c}4 \\
\text { optional } \\
\end{array}$ & $\$ 4,125,000$ & & & $\$ 4,125,000$ & & & $\$ 4,405,000$ & & $\$ 4,405,000$ & \\
\hline $\begin{array}{c}5 \\
\text { optional } \\
\end{array}$ & $\$ 70,000$ & & & $\$ 70,000$ & & & $\$ 60,000$ & & $\$ 60,000$ & \\
\hline Total & $\$ 4,195,000$ & & $\$ 185,543$ & $\$ 4,380,543$ & $\$ 200,385$ & $\$ 1,632,567$ & $\$ 4,465,000$ & & $\$ 1,297,952$ & \\
\hline
\end{tabular}




\section{0 - 2011 Tuition Differential Update}

Provide the following information for the 2010-2011 Academic Year.

\section{0-2011 - 70\% Initiatives (List the initiatives}

provided in the 2010-11 tuition differential request.)

Increase number of course sections.
University Update on Each Initiative

23 additional course sections were offered for students.

\section{Additional Detail, Where Applicable:}

Total Number of Faculty Hired or Retained (funded by tuition differential):

Total Number of Course Sections Added or Saved (funded by tuition differential):

2010-2011 - 30\% Initiatives (list the initiatives provided in the 2010-11 tuition differential request)

Increase the number of financial aid awards to undergraduate students with financial need.

Financial Aid awards are distributed and controlled by the USF System office.

\section{Additional Information (estimates as of April 30, 2011):}

Unduplicated Count of Students Receiving at least

one Tuition Differential-Funded Award:

Financial Aid awards are distributed and controlled by the USF System office.

\$ Mean (per student receiving an award) of Tuition

Differential-Funded Awards:

\$ Minimum (per student receiving an award) of

Tuition Differential-Funded Awards:

72

Maximum (per student receiving an award) of

Tuition Differential-Funded Awards:

$\$ 757$

$\$ 250$

$\$ 1,000$ 


\section{Fall 2011 Request for an Increased Tuition Differential Fee}

\section{University: POLYTECHNIC}

\section{Effective Date}

University Board of Trustees Approval Date:

Implementation date - August 2011

\section{Campus or Center Location}

Campus or Center Location to which the Tuition

Differential fee will apply (If the entire university, indicate as such):

\section{Undergraduate Course(s)}

Course(s). (If the tuition differential fee applies to all university undergraduate courses, indicate as such. If not, also provide a rationale for the differentiation among courses):

USF Polytechnic

The tuition differential will apply to all undergraduate courses offered by the USF System.

Current and Proposed Increase in the Tuition Differential Fee

Current Undergraduate Tuition Differential per credit hour:

Percentage tuition differential fee increase (calculated as a percentage of the sum of base tuition plus tuition differential):

$\$$ Increase in tuition differential per credit hour:

$\$$ Increase in tuition differential for 30 credit hours:

Projected Differential Revenue Generated and Intended Uses

Incremental differential fee revenue generated in

2011-12 (projected/calculated):

Total differential fee revenue generated in 2011-12

(projected/calculated):

\section{INSERT the following Documents:}

- Tuition Differential Schedule I (EXCEL)

- University Tuition, Fees, and Housing Projections (EXCEL)

- Legislative Budget Request (LBR) Summary (EXCEL)

- An Operating Budget (OB) Form I Narrative for each LBR Item (Word)

- Summary of the Five-Year Capital Improvement Plan(PECO and Challenge Grant )Projects (EXCEL) 


\section{STATE UNIVERSITY SYSTEM OF FLORIDA \\ Tuition Differential Collections, Expenditures, and Available Balances \\ University: USF POLYTECHNIC \\ Fiscal Year 2010-2011 and 2011-12}

University Tuition Differential

Budget Entity: 48900100 (Educational \& General)

SF/Fund: 2164xxx (Student and Other Fees Trust Fund)

Estimated Actual ${ }^{*}$

2010-11
Estimated

2011-12

\section{Balance Forward from Prior Periods}

\section{Balance Forward}

Less: Prior-Year Encumbrances

Beginning Balance Available:

Receipts / Revenues

Tuition Differential Collections

Interest Revenue - Current Year

Interest Revenue - From Carryforward Balance

Total Receipts / Revenues:

\section{Expenditures}

Salaries \& Benefits

Other Personal Services

Expenses

Operating Capital Outlay

Student Financial Assistance

Expended From Carryforward Balance

${ }^{* *}$ Other Category Expenditures

Total Expenditures:

Ending Balance Available:

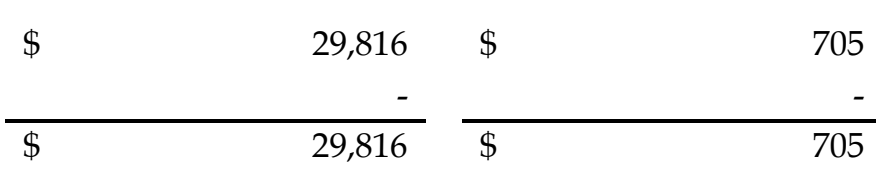

\$ 350,984

658,349

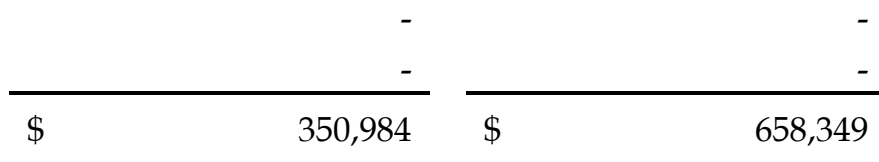

$\$$

$196,411 \quad \$$

259,742

40,333

201,200

114,240

197,717

29,111

$\begin{array}{lrr}\text { \$380,095 } & & - \\ & 658,659\end{array}$

\begin{tabular}{l}
\hline$\$ 05$ \\
\hline \hline
\end{tabular}

$\$$

*Since the 2010-11 year has not been completed, provide an estimated actual.

**Provide details for "Other Categories" used. 
REVISED 5-13-2011 


\section{State University System \\ Florida Board of Governors \\ Instructions for Completing the Revised Operating Budget (OB) Form I}

The OB Form I is designed to capture the data needed to align a university's operating budget issue with the goals and objectives of the State University System (SUS) Strategic Plan and the New Florida Initiative.

Each university should submit one sequential priority list of all budget issues for the university. Any issues unique to a branch campus or a special unit (e.g., IFAS, health science center) should be incorporated into the single university priority list, even if the university decides to separate the base allocation into prorated amounts for each branch campus or special unit.

For each budget issue, please indicate the primary goal from the SUS Strategic Plan that the issue will address, and complete the form according to the instructions provided.

Keep all responses brief. All issues must have been identified in the 2010 University Work Plan submitted to the Board of Governors and must align with the goals and objectives of the SUS Strategic Plan and the New Florida Initiative. 


\section{State University System \\ Education and General \\ 2011-2012 Legislative Operating Budget Issue \\ Form I}

\begin{tabular}{|l|}
\hline University: University of South Florida Polytechnic \\
Work Plan Issue Title: - STEM education and engineering; \\
interdisciplinary with business and innovation management, and \\
applied research in Alternative Energy and Biofuels Technologies
\end{tabular}

Although an issue might address multiple SUS Strategic Plan Goals, please check a single primary goal that this issue will address:

Access to and Production of Degrees (Examples of issues that might support this goal could include services such as outreach programs, new enrollment growth, new e-learning opportunities, or increased financial aid to improve student access; academic tracking, advising, tutoring, supplemental instruction, or other support services to improve undergraduate retention and graduation; or enhanced support to develop competitive recruitment packages for recruiting and retaining outstanding graduate and professional students.)

Meeting Statewide Professional and Workforce Needs (Examples of issues that might support this goal could include services that focus on the recruitment and retention of highly qualified students and faculty in disciplines associated with high-skill, high-wage jobs (e.g., STEM fields) or other areas of strategic emphasis in the State University System.)

\section{Building World-Class Academic Programs and Research Capacity (Examples} of issues that might support this goal could include focused support for academic programs on the cusp of national or international preeminence; support to achieve specialized accreditation in specific disciplines; new and/or expanded research initiatives built on the core strengths of the institution; or focused support to more quickly move cutting-edge university research to application and/or commercialization.)

\section{Meeting Community Needs and Fulfilling Unique Institutional}

Responsibilities (Examples could include issues important to a region or specific to an institution's mission - e.g., extension services, service learning initiatives, lifelong learning opportunities, community engagement initiatives, or targeted degree programs to meet regional needs.)

\section{Need and Justification:}


A. Identify the need as addressed explicitly in the $\mathbf{2 0 1 0}$ University Work

Plan, and indicate where this budget issue is referenced in the Plan.

USF Polytechnic requests funding to hire talented, competitive and experienced faculty to develop an Interdisciplinary Engineering degree program and to establish a Center for Applied Research in Alternative Energy and Biofuels Technologies. This goal is consistent with our vision of becoming a "premier destination campus for applied learning, research, and innovative technology" whose students and graduates "will inspire and lead change, locally and internationally."

Emphasis in faculty hiring is the identification of talented, competitive and experienced practitioner-scholars with capacity to deliver the polytechnic mission: interdisciplinary and applied learning; application of cutting-edge research and technology to real world needs; and collaborative partnerships that support economic, social and community development.

To enhance our capacity to move quickly to deliver new academic programs and expand research initiatives subsequent to separate SACS accreditation, we will be seeking faculty who have academic degrees from polytechnic or polytechnic-like universities or experience working in polytechnic or polytechnic-like universities.

This need is referenced in USF Polytechnic's 2010 Work Plan on pp. 7-8 under primary institutional goals and new academic degree programs.

B. Indicate how this budget issue aligns with the goal selected above from the SUS Strategic Plan.

This budget request aligns with the SUS Strategic Plan goal of building world-class academic programs and research capacity. Funding will provide opportunity to hire experienced faculty to develop a degree program and establish an applied research center that aligns with critical needs identified in the SUS Strategic Plan: STEM education and engineering, with potential further interdisciplinary opportunity in business and innovation management.

Talented, competitive and experienced faculty, prepared and/or experienced in the polytechnic model, will provide opportunity to move more quickly on the development of cutting-edge research application, technology transfer and/or commercialization. 
In addition, hiring talented, competitive and experienced faculty will enhance the university's ability to achieve specialized professional accreditation following separate SACS accreditation (e.g., ABET).

C. Indicate how this budget issue aligns with the objectives of the New Florida initiative.

The New Florida initiative focuses on the development of a knowledge and innovation economy built on high-technology and high-wage jobs in fields of science, technology, engineering and mathematics, medicine, finance, insurance, professional services, health care and education.

Building the New Florida requires new talent which includes not only increasing the percentage of Floridians who have baccalaureate and advanced degrees in these areas, but also bringing new competitive talent to the state to build new degree programs and research capacity in the State's universities.

The unique and specialized mission of the polytechnic in applied learning, research and innovative technology is well-aligned with the New Florida initiative. Hiring talented and competitive faculty, trained and/or experienced in the polytechnic model, will increase the university's ability to develop and deliver more quickly degree programs consistent with needs articulated in the New Florida initiative.

\section{Description:}

A. Description of service or program to be provided: (Include whether this is a new or expanded service/program. If expanded, what has been accomplished with the current service/program?)

While no new degree programs can be implemented until completion of SACS accreditation, new degree programs can be developed and taken through the USF System and State program approval processes for implementation after SACS accreditation. These new degree programs would be targeted for implementation in fall 2013 with the opening of the new campus site.

The development of a new degree program in Interdisciplinary Engineering not only aligns with the State's critical needs areas, but also with industry sectors identified by SRI International and Enterprise Florida as prime for future cultivation and growth. In addition, the program places emphasis on applied learning where students and faculty engage in interactive, problemand solution-based learning and development of applications of innovative research and technology to real-world problems. 
The establishment of a Center for Applied Research in Alternative Energy and Biofuels Technologies is consistent with the polytechnic model where students and faculty have world-class opportunities for interactive, problemand solution-based learning and for application of innovative research and technology. The Center will also provide students with opportunity for participation in a dynamic learning community, a collaborative learning lab, and field experiences and internships

USF Polytechnic will use these funds to attract, recruit and support the teaching and research needs of talented and competitive new faculty, as well as establish a recurring funding base to operate the Center.

\section{B. Description of current university initiatives and resources that will strengthen the provision of this service or program:}

Goal 3 of the USF Polytechnic Strategic Plan 2007-2012 established the university's direction in the expansion and/or creation of academic programs that focus on applied learning, applied research, applied technology, and interdisciplinary approaches in a polytechnic model.

The structure of USF Polytechnic's colleges accommodates its existing degree programs, allows for the development of these new degrees, and reflects commitment to interdisciplinary learning and research engagement. The new faculty and degree program will be housed in the College of Technology and Innovation which comprises the Divisions of Innovation Management, Engineering \& Applied Sciences, and Information Technology. The Center will be housed in the High Tech Research, Innovation and Business Incubator \& Learning Labs, one of the first buildings planned for the new I-4 campus.

Current university resources have been used to hire faculty and staff to support existing degree programs and meet SACS accreditation requirements, including expanding faculty in Innovation Management and Industrial Engineering. Both fields offer potential for further interdisciplinary academic and research opportunities in conjunction with the development of an Interdisciplinary Engineering degree program and the Center for Applied Research in Alternative Energy and Biofuels Technologies.

C. Description of outcome(s) anticipated or dashboard indicator(s) to be improved: (Be specific. For example, if this issue focuses on improving retention rates, indicate the current retention rate and the expected increase in the retention rate. In addition, identify the following, if applicable.)

1. Number of Headcount Students receiving services or participating in the program by year, for the next five years: 


\begin{tabular}{|c|c|c|c|c|}
\hline 2011-2012 & $2012-2013$ & $2013-2014$ & $2014-2015$ & $2015-2016$ \\
\hline NA & NA & 25 & 50 & 75 \\
\hline
\end{tabular}

2. Number of FTE Students receiving services or participating in the program by year for the next five years:

\begin{tabular}{|c|c|c|c|c|}
\hline 2011-2012 & $2012-2013$ & $2013-2014$ & $2014-2015$ & $2015-2016$ \\
\hline NA & NA & 10 & 20 & 30 \\
\hline
\end{tabular}

3. Additional degrees, if any, produced as a result of this initiative: (Indicate the additional number of Bachelor's, Master's, Doctoral, \& Professional degrees to be produced by school year.)

B.S. Interdisciplinary Engineering - 2013

M.S. Energy and Environmental System Engineering - 2016

\section{Other outcomes:}

Critical to successful completion of initial SACS accreditation is the identification and implementation of a faculty and staff hiring plan to build depth in existing faculty, capacity for delivery of existing degree programs, and additional support for student services. As accreditation is achieved, faculty and staff hiring plans will continue to focus on capacity for delivery of the new "polytechnic" degrees and additional capacity for full implementation of general education offerings for freshman and sophomore classes in fall 2013.

Faculty hired through this funding request will also contribute to the development and delivery of a General Education core, focusing on a narrow number of course offerings, aligned with the USF Polytechnic Core Values as identified in the 2007-2012 Strategic Plan. We seek faculty who can teach in both a primary and secondary content area. This will enable USFP to deliver general education that meets State requirements, demonstrates measurable performance-based competencies, and includes field-based and internship experiences for all students with fewer course offerings.

The establishment of a Center for the Development of Alternative Energy and Biofuels Technologies will provide increased opportunity to seek grants and contracts to further enhance research capacity. 


\section{Facilities:}

A. Does this issue require an expansion or construction of a facility?

YES

B. If yes, is the project identified on the Capital Improvement List? If so, identify the project, fiscal amount, year requested, and priority number.

The Center for Applied Research in Alternative Energy and Biofuels Technologies will be one of the entities initially housed in the High Tech Research, Innovation and Business Incubator \& Learning Labs. Additional private funding will be sought to expand facilities for the Center.

\begin{tabular}{|c|l|c|c|}
\hline \multicolumn{1}{|c|}{ Facility Project Title } & Fiscal Year & Amount Requested \\
\hline 6. & $\begin{array}{l}\text { USF Health School of } \\
\text { Pharmacy at USF Polytechnic }\end{array}$ & $2011-2012$ & $\$ 10,000,000$ \\
\hline 21. & $\begin{array}{l}\text { USF Polytechnic I-4 Campus } \\
\text { Phase IIA High Tech } \\
\text { Research, Innovation and } \\
\text { Business Incubator \& } \\
\text { Learning Labs - FECG }\end{array}$ & $2011-2012$ & $\$ 700,000$ \\
\hline
\end{tabular}

In addition, a PECO request has been made for the High Tech Research, Innovation and Business Incubator \& Learning Labs in the amount of $\$ 10,000,000$. It is not on the current five-year CIP listing. 